RECYT

Año 22 / № 33 / 2020 / 63-75

\title{
Entre las curvas de la arquitectura contemporánea y la enseñanza de la geometría en arquitectura: un enfoque didáctico del diseño paramétrico
}

\author{
Between the curves of contemporary architecture and the teaching of geometry in \\ architecture: a didactics approach to the parametric design
}

\section{Entre as curvas da arquitetura contemporânea e o ensino da geometria na arquitetura: uma abordagem didática ao projeto paramétrico}

\author{
Janice de Freitas Pires ${ }^{1,{ }^{*}}$, Alice Theresinha Cybis Pereira ${ }^{1}$ \\ 1- Universidade Federal de Pelotas, Brasil. \\ *E-mail: janicefpires@hotmail.com \\ Recibido el 25 de agosto de 2019, Aprobado el 18 de diciembre de 2019.
}

\section{Resumen}

La arquitectura contemporánea experimenta momentos de gran transformación, especialmente en relación con el proceso de diseño, el uso de materiales de construcción innovadores y técnicas y simulación, con el objetivo de evaluar el rendimiento del objeto arquitectónico a lo largo del proceso, desde la concepción hasta su vida útil. En este contexto, identificamos la presencia de nuevos conocimientos que deben ser apropiados por arquitectos y diseñadores, ya que estos son los principales involucrados en todo el proceso (concepción, diseño, construcción, evaluación de uso). En consecuencia, existe un problema didáctico para las escuelas de arquitectura, en el sentido de preparar a estos profesionales para trabajar en el mercado laboral de proyectos contemporáneos. Uno de los enfoques involucrados en tales procesos de diseño contemporáneo se relaciona con la complejidad geométrica de las formas propuestas, que se definen en base a un conjunto de enfoques. Estos pueden ser aquellos basados en el desarrollo tecnológico que permite integrar entornos digitales de representación gráfica y simulación para la búsqueda de formas y su optimización y, por otro lado, la exploración del funcionamiento de los sistemas naturales, sus procesos y la formación de sus geometrías, aplicadas por Gaudí, Fray Otto, Félix Candela, Heinz Isler y Luig Nervi en el pasado. La conexión entre las prácticas de diseño computacional y los fenómenos de la naturaleza surge como un enfoque potente de la arquitectura en el sentido de la economía de los materiales y la integración cualitativa con el entorno o lugar de inserción. Anteriormente, a través de una investigación doctoral, se constituyó una red de conceptos de las superficies complejas de la arquitectura contemporánea, y, en el presente estudio, el objetivo, a partir de un caso de arquitectura referencial, es promover una investigación didáctica, con el objetivo de construir referencias para la adopción de técnicas de modelado paramétrico y fabricación digital de tales superficies, como soporte para la acción de diseño.

Palabras clave: arquitectura contemporánea, geometría compleja, modelado paramétrico, superficies mínimas, enseñanza de arquitectura.

\section{Abstract}

Contemporary architecture is undergoing great transformational moments, especially concerning with the process design, the use of innovative construction materials and techniques, and simulation, which aim at evaluating the performance of the architectural object throughout the whole process: from conception to useful life. In this new context, we identify updated knowledge that needs to be appropriated by architects and designers, who are the main participants of the whole process (conception, design, construction, evaluation and use). Consequently, there is a didactic problem within the schools of architecture because they teach professionals to work for the labor market with contemporary projects. One of the approaches involved in the contemporary design processes is related to the geometric complexity of the proposed shapes, defined and based on a set of approaches. On the one hand, such approaches can be those which are based on technological development that allows integrating graphic representation and simulation for the digital environment with the purpose of the shape searching and its optimization and; on the other hand, the exploration of the natural system functioning, its process and the 
geometrical formation, as the ones implemented by Gaudí, Friar Otto, Félix Candela, Heinz Isler and Luig Nervi. The connection between computational design practices and nature phenomena emerges as a potent approach for architecture regarding with material savings and its qualitative integration with the environment or insertion place. A network of concepts about the complex surfaces of contemporary architecture had previously been established thanks to a doctoral research. Considering that architecture referential case, the objective of the present study is to promote a didactic investigation on the use of complex surfaces in architecture, which aims at building up new references for both the adoption of parametric modeling techniques and such digital fabrication surfaces as a support for the action design.

Keywords: contemporary architecture, complex geometry, parametric modeling, minimal surfaces, architecture teaching.

\section{Resumo}

A arquitetura contemporânea vive momentos de grandes transformações, relativas especialmente ao processo de projetar, ao emprego de materiais e técnicas de construção inovadoras e de simulação, visando avaliar o desempenho do objeto arquitetônico durante todo o processo, desde a concepção até a sua vida útil. Neste contexto, identificam-se a presença de novos saberes os quais necessitam serem apropriados pelos arquitetos e designers, desde que estes são os principais envolvidos em todo o processo (concepção, projeto, construção, avaliação de uso). Consequentemente, configura-se um problema didático para as escolas de arquitetura, no sentido de preparar tais profissionais para atuação no mercado de trabalho do projeto contemporâneo. Uma das abordagens envolvidas em tais processos de projeto contemporâneo relaciona-se com a complexidade geométrica das formas propostas, as quais são definidas com base em um conjunto de abordagens. Estas podem ser desde as que se fundamentam no desenvolvimento tecnológico que possibilita integrar ambientes digitais de representação gráfica e simulação para a busca da forma e a sua otimização e, por outro lado, na exploração do funcionamento dos sistemas naturais, seus processos e a formação de suas geometrias, tal como aplicado por Gaudì, Frei Otto, Félix Candela, Heinz Isler e Luig Nervi no passado. A conexão entre práticas de projeto computacional e fenômenos da natureza surge como uma abordagem potente na arquitetura, no sentido de economia de materiais e integração qualitativa com o ambiente ou o lugar de sua inserção. Anteriormente, por meio de uma pesquisa de doutorado, foi constituída uma rede de conceitos das superfícies complexas da arquitetura contemporânea, e, no estudo presente, objetiva-se, a partir de um caso de arquitetura referencial, promover uma investigação didática sobre o emprego de superfícies complexas na arquitetura, visando à construção de referenciais para a adoção de técnicas de modelagem paramétrica e fabricação digital, como suporte à ação projetual.

Palavras-chave: arquitetura contemporânea, geometria complexa, modelagem paramétrica, superfícies mínimas, ensino de arquitetura.

\section{Introdução}

A arquitetura contemporânea vive momentos de grandes transformações, relativas especialmente ao processo de projetar, ao emprego de materiais e técnicas de construção inovadoras e de simulação, visando avaliar o desempenho do objeto arquitetônico durante todo o processo, desde a concepção até a sua vida útil. Neste contexto, identificamse a presença de novos saberes os quais necessitam serem apropriados pelos arquitetos e designers, desde que estes são os principais envolvidos em todo o processo de projeto e construção.

Consequentemente, configura-se um problema didático para as escolas de arquitetura, no sentido de preparar tais profissionais para atuação no mercado de trabalho do projeto contemporâneo. Tal problema passa pela conscientização e conhecimento sobre as formas criadas em tais processos projetuais, apontando para a inclusão nos currículos das faculdades de arquitetura de propostas didáticas que possibilitem uma ampla interação entre os fundamentos arquitetônicos presentes em tais geometrias complexas, relativos aos seus elementos teóricos, as técnicas e tecnologias de representação gráfica (como a modelagem paramétrica e a fabricação digital). No entanto, tais conteúdos são muito pouco abordados nos contextos de ensino de arquitetura no Brasil. O conhecimento sobre a geometria ainda é tratada de maneira restrita em abordagens de seus aspectos representacionais advindos da geometria descritiva, em que prevalece a aplicação do sistema bi projetivo para o desenho. Identifica-se a necessidade de um enfoque didático para a inserção destes novos conceitos na arquitetura, por sua característica interdisciplinar (geometria complexa, projeto, computação) e o qual possa ser integrado aos diferentes métodos adotados nas disciplinas de projeto e de representação.

Uma das abordagens envolvidas em tais processos de projeto contemporâneo relaciona-se com a complexidade geométrica das formas propostas, as quais são definidas com base em um conjunto de abordagens. Estas podem ser desde as que se fundamentam no desenvolvimento 
tecnológico que possibilita integrar ambientes digitais de representação gráfica e simulação para a busca da forma e a sua otimização e, por outro lado, na exploração do funcionamento dos sistemas naturais, seus processos e a formação de suas geometrias, tal como aplicado por Gaudì, Frei Otto, Félix Candela, Heinz Isler e Luig Nervi no passado [1] [2] [3]. A conexão entre práticas de projeto computacional e fenômenos da natureza surge como uma abordagem potente na arquitetura, no sentido de economia de materiais e integração qualitativa com o ambiente ou o lugar de sua inserção.

No contexto em que este trabalho se insere, foi constituída uma rede de conceitos das superfícies complexas da arquitetura contemporânea, a qual objetivou apoiar o reconhecimento dos aspectos teóricos e tecnológicos para a representação gráfica digital de tais geometrias empregadas na arquitetura [4]. Para o desenvolvimento de tal rede de conceitos, delimitou-se um processo de análise de saber, de acordo com os termos da Teoria Antropológica da Didática [5]. O pressuposto foi de que a explicitação deste 'saber' oferecia uma base de conceitos para subsidiar processos de aprendizagem de modelagem paramétrica [6] aplicada à geração de geometrias complexas da arquitetura contemporânea.

No estudo presente, objetiva-se, a partir de um caso de arquitetura referencial, configurada por uma superfície mínima [2] [7], promover uma investigação didática sobre o emprego de superfícies complexas na arquitetura, visando à construção de referenciais para a adoção de técnicas de modelagem paramétrica e fabricação digital, como suporte à ação projetual. A relevância deste estudo está em contribuir ao conhecimento sobre a aplicabilidade de tais geometrias e ir além dos aspectos técnicos da representação e usar a representação (modelagem) para conhecer aspectos funcionais e qualitativos de tais geometrias.

\section{Referenciais teóricos e metodológicos}

Preliminarmente, foi identificado que existem iniciativas em universidades do exterior e do Brasil de aplicação das técnicas de modelagem paramétrica no ensino do projeto de arquitetura, geralmente utilizando métodos focados em oficinas denominadas de workshops. Estes investem predominantemente nos aspectos tecnológicos para a resolução de várias classes de problemas arquitetônicos, com abordagem pouco abrangente sobre a interdependência entre as teorias e técnicas que suportam este conhecimento [4]. Principalmente quanto à explicitação da relação entre estrutura geométrica e qualidades arquitetônicas das geometrias complexas, como conhecimento de projeto. Dessa maneira, investe-se no reconhecimento dos elementos de saber envolvidos em tais processos de ensino, nos termos da teoria didática de Chevallard [5], a partir de um modelo de análise que esta teoria disponibiliza. Este modelo identifica a presença, na ação humana, de 04 elementos principais os quais forma a sua praxeologia (sua estrutura lógica): os problemas ou tarefas, as técnicas de resolução das tarefas, as tecnologias que justificam, explicam e produzem as técnicas, e as teorias, que possuem o mesmo papel em relação às tecnologias, de explicação, justificação e produção. Segundo o mesmo autor, ao se desenharem atividades didáticas, é importante que estas veiculem o saber em sua estrutura integral, sendo necessário para isto que os seus elementos estejam explicitados e selecionados previamente.

Segundo Scott Chase [8], pág. 662, uma análise visando à adoção de práticas didáticas nas escolas de arquitetura a partir do uso de ferramentas computacionais, passa pela questão chave da integração

A integração é a palavra-chave para o desenvolvimento de novos currículos do CAAD. Dada à pedagogia arquitetônica tradicional, a implementação pode ser difícil. Um dos principais objetivos é permitir que os alunos vejam a relevância da computação para o processo de design e assegurem que ela seja utilizada adequadamente

$\mathrm{O}$ autor se refere à abordagem tradicional da representação e do desenho no ensino de arquitetura que pouco investe na integração entre ferramentas computacionais e processo de projeto, no sentido de exploração destas para a ação projetual em si. Nesse contexto, a adoção nos últimos anos de iniciativas em projeto paramétrico veio desafiar os docentes para a conscientização da necessidade de novas estratégiad didáticas, que possibilitem tal integração entre vários campos de conhecimento.

Marc Aurel Schnabel [8] pontua que "a educação do CAAD tem que estimular o interesse no projeto arquitetônico e, mais importante, estabelecer um mecanismo para controlar e melhorar a qualidade do projeto produzido com a ajuda da mídia digital" (pág. 664). Modelos e escaneamentos tridimensionais, ambientes virtuais e prrototipagem rápida são usados no atelier para auxiliar estudantes e professores a explorar e estudar a criatividade arquitetônica de forma a permitir um envolvimento mais profundo em questões de design.

Pode-se concluir que o autor se refere à manipulação de modelos direcionada a predição e avaliação realizada computacionalmente durante o processo de projeto, integrada uma base teórica que o projetista precisa conhecer.

Kvan [8], pág. 667, destaca, neste contexto, “o papel da arquitetura como conhecimento, em que os docentes poderiam ver a maneira de ensinar como recolha e manipulação deste conhecimento".

Em todas estas abordagens pode se reconhecer a preocupação de explicitar o elevado grau de complexidade que o projeto de arquitetura incorpora paralelamente com a apropriação de tais tecnologias pelos estudantes.

Kvan et al.. [9] destacam que, em vez de um ensino focado em um conjunto de técnicas de modelagem e de renderização, deveriam ser aplicadas atividades estratégicas que demonstrem o impacto de tais técnicas para a 
compreensão de como os projetos podem ser visualizados e processados para suportar o pensamento de projeto.

Os mesmos autores também destacam que, se por um lado estas atividades aumentam a criatividade e a intuição através de ambiguidades e abstrações, por outro lado, capturam e controlam a complexidade, além de proporcionar precisão dimensional baseada em materiais físicos reais.

Para os autores, no momento em que os estudantes avançam para os níveis superiores de um programa de projeto, sua capacidade de aproveitar essas técnicas pode crescer em complexidade com a natureza dos problemas de design que emergem. Os autores exemplificam que uma maior sofisticação na modelagem e na análise geométrica pode acompanhar a resolução de problemas de projeto mais articulados e tectonicamente avançados no atelier de projeto. Outra questão a destacar negativamente é o não incentivo a exercitar habilidades de pensamento tridimensional da geometria descritiva, e perceber os meios de projeto digital como dispositivos unicamente de representação.

\section{Materiais e métodos}

O presente estudo tem um enfoque didático amparado nas teorias de Chevallard: a Teoria da Transposição Didática [10] e a Teoria Antropológica da Didática [4]. Conforme já mencionado, este autor destaca os elementos de um saber que devem ser considerados em processos de ensino e aprendizagem, com vistas a uma transposição didática de tal saber. Tendo por base tais teorias, identificou-se que a natureza descritiva da modelagem algorítmica, que exige reconhecer elementos teóricos, técnicos e tecnológicos, pode potencializar a explicitação do saber da geometria complexa das estruturas regenerativas empregadas na arquitetura. $\mathrm{O}$ reconhecimento de processos de modelagem paramétrica desenvolvida em linguagem de programação visual por meio do plug-in Grasshopper junto ao software Rhinoceros, ao integrar a linguagem algorítmica em uma abordagem descritiva e visual, pode ser considerado como uma estratégia didática no ensino de arquitetura.

A modelagem paramétrica introduz também maiores possibilidades para a definição de geometrias complexas, a geração de instâncias de projeto e a avaliação destas instâncias, por ser um processo de representação baseado em um sistema que armazena todos os dados relacionados à geometria do objeto que está sendo criado e representado e permite fazer relações entre estes dados. A escolha pela modelagem com linguagem de programação visual e descritiva também se justifica pelo fato de que ainda não se conta nos cursos de arquitetura com a inserção da linguagem pura de programação, exclusivamente por meio do uso de scripts.

A finalidade do reconhecimento de uma estrutura integral do saber que envolve tais geometrias é dar subsídios aos próprios docentes para a estruturação de situações didáticas e aos estudantes para tornarem-se conscientes de suas escolhas e proposições projetuais, além do uso adequado e não de modo gratuito da liberdade formal no projeto de arquitetura.

Dessa maneira, o estudo refere-se às etapas de reconhecimento e explicitação das estruturas de saber que envolvem a geometria complexa da arquitetura contemporânea, a partir do estudo de casos referenciais, como os que empregam superfícies mínimas na arquitetura e a proposição de processos de modelagem paramétrica destas. Para este trabalho em particular, foram desenvolvidas as seguintes etapas:

1. Seleção de uma obra de arquitetura com geometria de superfície mínima obtida por meios físicos. A obra selecionada foi identificada nos estudos de Burry \& Burry [11], os quais sistematizaram um conjunto de conceitos matemáticos extraídos de obras da arquitetura contemporânea;

2. Reconhecimento dos discursos que explicam a obra a partir de descrições da sua geometria. Nesta etapa, com o objetivo de reconhecer a obra em seus aspectos geométricos e arquitetônicos, analisaram-se os discursos de Burry $\&$ Burry [11] que descrevem um conjunto de obras de arquitetura a partir dos conceitos matemáticos subjacentes a suas geometrias. Estes autores apresentam tais conceitos articulando-os com os atributos arquitetônicos em termos formais e de desempenho, visando fundamentar tais propriedades das formas geométricas das obras estudadas.

3. Explicitação e ampliação dos conceitos reconhecidos nos estudos de Burry \& Burry. Nesta etapa, com base na Teoria Antropológica da Didática [4], buscou-se explicitar, a partir de autores advindos da Matemática, da geometria descritiva e do campo da Física, as estruturas de saber envolvidas em tais geometrias e nos conceitos apresentados pelos autores anteriormente citados;

4. Estruturação de processos de modelagem paramétrica de tais superfícies, por meio da linguagem de programação visual, disponibilizada pelo plug-in Grasshopper junto ao software Rhinoceros.

5. Estruturação de uma rede de conceitos que abarca a estrutura integral do saber relacionado às superfícies mínimas empregadas na obra Main Station Stuttgart. Tal estrutura de saber envolve as teorias subjacentes às superfícies mínimas (que possibilitam explica-las do ponto de vista tecnológico), as técnicas de modelagem de tais superfícies e as tecnologias (que incorporam discursos racionais sobre as técnicas de modelagem empregadas, justificando-as e explicando-as).

\section{Análise da estrutura de saber que envolve a geometria do Main Station Stuttgart}

A obra selecionada para o estudo em questão é o Main Station Stuttgart, de Igenhoven Architects (Figura 1), configurada por uma superfície mínima [5] obtida fisicamente por meio de modelos em suspensão [2] [3]. Na obra, 
esta superfície foi utilizada com o objetivo de configurar espaços contínuos e, ao mesmo tempo, obter uma solução de otimização estrutural por intermédio do uso de uma casca de concreto no formato de um 'cálice' (modelo de referência do arquiteto alemão Frei Otto, 1966).

Uma análise preliminar sobre a obra e a superfície empregada é dada em Burry \& Burry [11], por meio de descrições textuais. Na sequência, é adotado o método de ampliação das estruturas de saber identificadas em tais descrições, a partir de autores específicos da matemática e engenharia. Logo, estruturam-se os processos de modelagem paramétrica da superfície e discute-se qual o potencial destes para o ensino de arquitetura.

No enfoque da teoria didática adotada, o reconhecimento de estruturas de saber é uma etapa prévia a estruturação de situações e materiais didáticos, válida para qualquer contexto educativo, o que justifica o método adotado neste estudo.

\section{A caracterização de uma obra de arquitetura com superfície mínima}

A construção da obra Main Station Stuttgart abrangerá plataformas ferroviárias de 420 metros de comprimento e conectará as zonas urbanas de pedestres com o histórico parque Schlossgarten (Figura 1, à direita). Sua geometria estrutural (Figura 1, à esquerda) foi desenvolvida em estreita colaboração interdisciplinar entre o escritório de arquitetura, engenheiros civis e em conjunto com Frei Otto. A estrutura é uma casca contínua, formada por 28 orifícios, que proporcionam luz e ventilação natural na estação de metrô. Além disso, o tipo de geometria da cobertura da estação é de carga altamente eficiente, atuando apenas em compressão. Os aspectos de sustentabilidade também foram pensados no projeto da obra: é uma estação de energia zero, em que está previsto quase nenhum consumo de energia primária. Aquecimento, arrefecimento e iluminação serão todos naturais. Para o arquiteto, neste projeto, o objetivo de traduzir a nova era no transporte ferroviário em uma forma contemporânea foi alcançado pelas qualidades de engenharia interdisciplinar e virtudes de design [12].

\section{As descrições sobre a obra por autores que analisam arquitetura}

A proposta da nova estação integra o plano diretor de Stuttgart 21, sendo um edifício disposto a $90^{\circ}$ da estação existente, orientado para novos tuneis ferroviários. O seu salão monumental seria construído abaixo do solo, para que parte deste fosse utlizado pela população, com espaços verdes [11].

O âmago do projeto é o emprego de uma superfície mínima encontrada fisicamente, mas que também é descrita e compreendida matematicamente, com o propósito de abar-
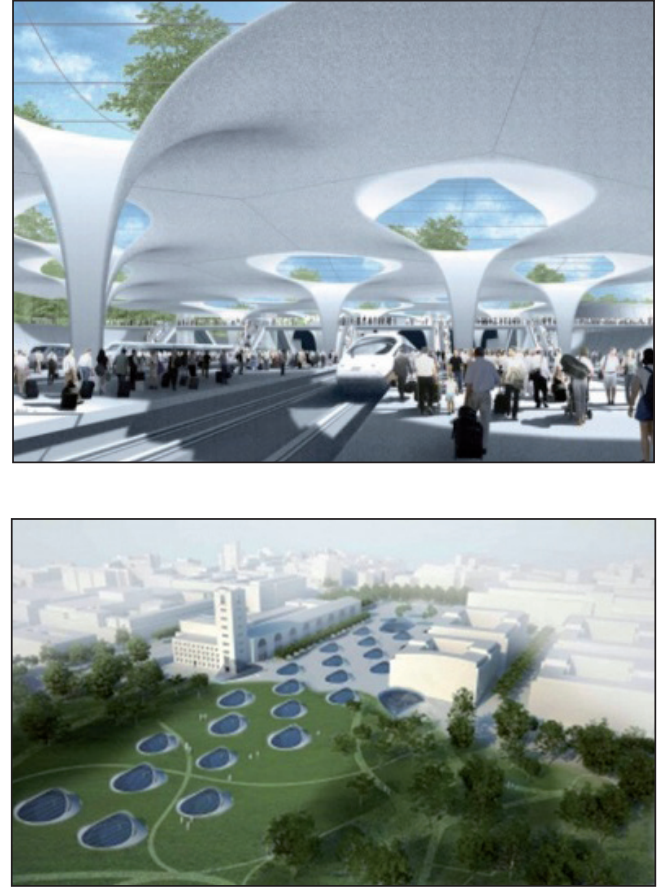

Figura 1: Main Station Stuttgart, projeto Ingenhoven Architects. Fonte das imagens: https://www.lafargeholcim-foundation.org/ projects/main-station-stuttgart-germany http://www.bahnprojektstuttgart-ulm.de/en/details/new-stations/stuttgart-main-station/architecture/

car simultaneamente dois problemas: a economia estrutural e material; e a provisão de luz e ar para o enorme espaço subterrâneo, sem criar consumo de energia significativo ou poluição de carbolno [11]. A dimensão do salão principal é de $420 \mathrm{~m} \times 80 \mathrm{~m}$, podendo abrigar até 300 mil passageiros.

O tipo de superfície mínima da obra se forma em torno de um orifício (denominado de 'olho'), no qual existe um fluxo suave de tensões de tração. A superfície mínima se conforma em uma unidade protótipa com o formato de um funil e cálice, que possui a função combinada de telhado, suporte vertical e abertura para o céu [11].

\section{A explicitação da estrutura de saber}

A superfície mínima descrita no relato anterior é conformada por meio de modelos deformáveis que são submetidos a forças de tensão e relaxamento, denominados de 'modelos de catenária'. Tecnicamente, uma superfície mínima é aquela que possui valor de curvatura total oposta igual à zero em todos os seus pontos [11], obtendo equilíbrio estável [7].

Existem duas superfícies curvas tradicionais que também são mínimas: o helicoide e o catenoide (Figura 2), os primeiros exemplos, exceto o plano, encontrados pelos matemáticos, no século XVIII, logo após os primeiros estudos de Lagrange relacionados a problemas com superfícies mínimas, em 1760, mesmo ano em que Euler estabeleceu o conceito de curvaturas [7].

O catenoide é gerado pela varredura de uma curva catenária ao longo de um eixo e o helicoide é gerado pela rotação e translação simultânea de uma linha [13], as duas 
superfícies ilustradas a esquerda da Figura 2. Outros exemplos descobertos matematicamente incluem as superfícies de Enneper, em 1864, e a recente superfície de Costa (à direita da Figura 2), em 1982, matemático brasileiro que, com seus estudos, veio a impulsionar, no século XX, o avanço da teoria de superfícies mínimas [7].

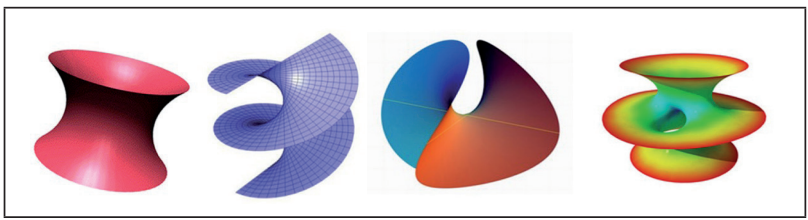

Figura 2: Superfícies mínimas encontradas matematicamente, da esquerda para a direita: Catenoide, Helicoide, Superfície de Enneper e Superfície de Costa.

Fonte das imagens: https://www2.le.ac.uk/departments/mathematics/extranet/staff-material/staff-profiles/k196/stuff/lopez-rosdeformation-of-the-catenoid/view; http://www.daviddarling.info/ encyclopedia/H/helicoid.html; http://www.indiana.edu/ minimal/maze/ enneper.html; http://www.eg-models.de/models/

Experimentos com bolhas de sabão, desenvolvidos por Plateau, no século XIX, inspiraram arquitetos do século $\mathrm{XX}$ ao estudo com modelos físicos de superfícies mínimas, denominados de 'modelos de catenárias' [11]. As catenárias são curvas que se configuram a partir de cabos ou hastes deformados sob a ação da gravidade, gerando uma forma (arco) que atua em pura compressão (Figura 3), da mesma maneira que uma superfície mínima. Os modelos e estruturas que se configuram a partir deste conceito são também denominados de 'modelos ou estruturas funiculares' [2] [3].

Conceitualmente, uma estrutura funicular pode ser definida como uma estrutura que pode alcançar o estado de equilíbrio adotando um mecanismo de forma "correta" (forma / geometria) correspondente às cargas aplicadas [14]. Essa forma "correta" é referida como a "geometria funicular". A geometria funicular é, portanto, a geometria de uma estrutura funicular [14]. Perez e Garcia [2], citando trabalhos de Frei Otto com superfícies mínimas, referem-se às estruturas funiculares encontradas na natureza e entre estas destacam as conchas e tendas como princípios estruturais que são aplicados na arquitetura. Para estes autores, ambas são estruturas de forma ativa por terem a capacidade de carga por sua configuração espacial, trabalhando em regime axial.

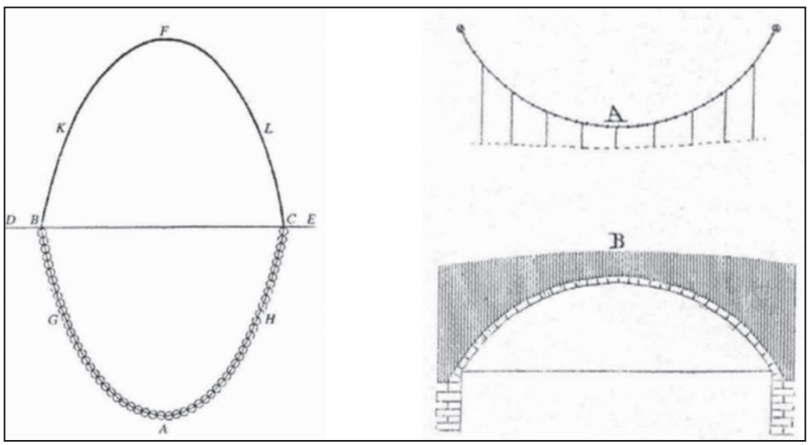

Figura 3: Arcos Catenários aplicados em pontes. Fonte: A. desenhado por Poleni 1748 in HUERTA (2006, pág. 325); B. Young 1807 in HUERTA (2006).
A idealização de Hooke, em 1671, sobre arcos estáveis, obtidos a partir da curva catenária, levou diretamente ao uso de modelos simples pendurados para projetar e calcular [15]. Isto pode ser exemplificado pelos arcos que os engenheiros ingleses do século XIX, notavelmente John Robison, construíram fazendo uso desta técnica; outro exemplo apontado pelo autor é o de modelos pendurados para dimensionar os contrafortes de uma igreja na Alemanha utilizados por Hübsch [15].

No final do século XIX e início do século XX, Gaudí utilizou este método para configurar seus arcos e estruturas funiculares, especialmente para as obras Colônia Güell e Sagrada Família. Huerta (pág. 325) reitera que Gaudí, no entanto, fez uso do conceito de arcos catenários de uma forma totalmente original: no sentido de integrar o projeto estrutural no processo de projeto arquitetônico [15]. Não se tratava de verificar a estabilidade de um determinado desenho, e sim de projetar desde o início usando formas estáveis. O autor ressalta que "Tanto quanto sabemos, é a primeira vez que essa tentativa é feita e explorada a toda a sua capacidade" [15].

Os modelos de suspensão (Figura 4), além de terem sido utilizados na arquitetura por Gaudi, no processo de design da Cripta de Colònia Güell associados a métodos gráficos como ferramentas de design [3], também foram usados no século XX por Frei Otto e sua equipe para encontrar a forma para o Mannheim gridshell e Heinz Isler, que desenhou suas conchas de concreto com base em modelos de pano pendurado [16] [17].
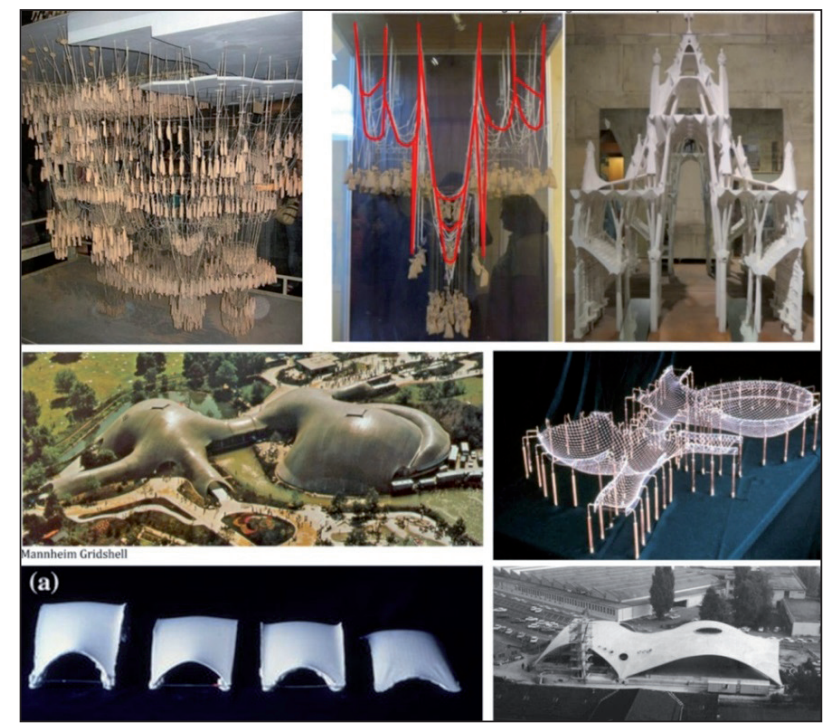

Figura 4: Modelos funiculares empregados na arquitetura nos séculos XIX-XX: na linha superior, modelos de Gaudì para a Colonia Güell (à esquerda) e Sagrada Família (ao centro e à direita); na linha central, modelo de Frei Otto para o Mannheim Gridshell; e na linha inferior, modelos de pano de Heinz Isler. Fonte das imagens: https://www.quora. com/What-is-Funicular-geometry-What-is-its-significance-in-Structuresin-Architecture; https://www.sciencedirect.com/science/article/pii/ S2214399815300011; Chilton e Chuang [17].

Para o projeto da estrutura da obra do Main Station Sttugart, Ingenhoven Architects desenvolveram um processo integrador, com muitos especialistas em planejamento e procedimentos. O principal objetivo construtivo era de 
minimizar a altura da estrutura do telhado enquanto seria alcançada uma maximização do espaço livre interno do hall da estação. Com este fim, foram incorporados os resultados dos trabalhos em curso desde o ano de 1963 no Institute for Lightweight Shell Structures (Institut für leichte Flächentragwerke da Universidade de Stuttgart), de Frei Otto, em que as membranas de películas de sabão foram utilizadas para desenvolver superfícies com uma distribuição completamente uniforme de tensões, conformando superfícies mínimas (Figura 5).

Ainda segundo os arquitetos da obra, em uma abordagem experimental, o módulo individual (em formato de olho e funil) juntamente com os suportes do funil e as paredes de calha foram desenvolvidos em modelos reais de corrente suspensa (Figura 6).

Em paralelo, houve um processo de busca de formas utilizando programas digitais com tecnologia CAD de superfícies de área mínima e os resultados de ambos os modelos foram comparados, para a configuração final do modelo de volume tridimensional do hall da estação. A geometria de dupla curvatura foi, ainda, otimizada e refinada a partir de cálculos baseados em elementos finitos ${ }^{1}$ (ROK OFFICE, http://www. rok-office.com/projects/0990-stuttgart-21-parametric-fem/). Com esse fim, foram desenvolvidos algoritmos específicos para gerar modelos complexos de elementos finitos para análise estrutural, os quais puderam ser implementados em sotware CAD como ferramentas flexíveis, para gerar e modificar modelos complexos de malha.

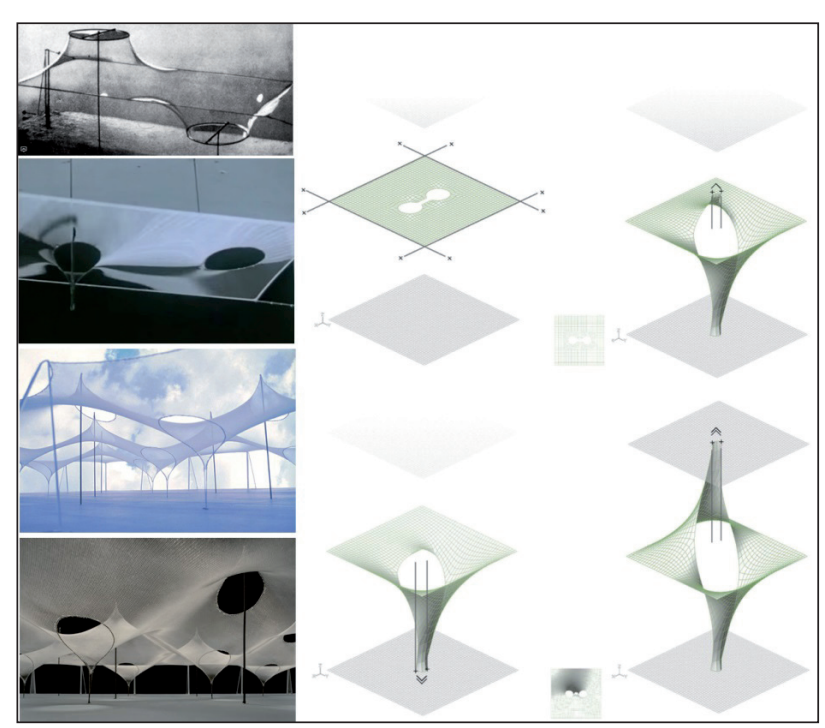

Figura 5: - Modelos de superfície mínima e Modelo funicular de Frei Otto.

Fonte: https://www.archdaily.com.br/br/763770/pritzker-2015-freiotto-e-a-importancia-da-experimentacao-na-rquitetura?ad

medium=widget\&ad_name=navigation-prev; https://www.archdaily com.br/br/763720/video-frei-otto-experimentando-com-bolhas-desabao; https://archpaper.com/2015/05/frei-otto-1925-2015/; http:// www.bmiaa.com/frei-otto-thinking-in-models-the-biggest-exhibitionever-on-the-german-master/; https://parametricsemiology.wordpress. com/2013/10/17/grp_06-session_01/

1- O Método dos Elementos Finitos (MEF) (Finite Element Method - FEM) é um procedimento numérico para determinar soluções aproximadas de problemas complexos, em que o domínio de um problema é subdividido em partes menores, denominadas de elementos finitos.
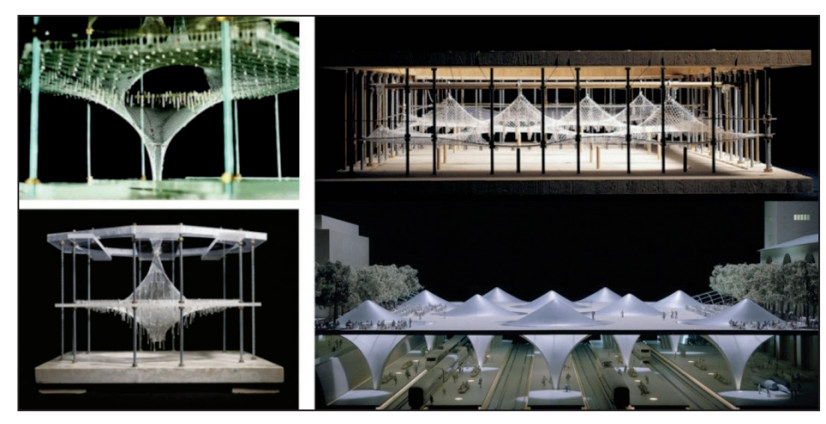

Figura 6: Modelo funicular (de corrente de suspensão) proposto para o Main Station Stuttgart.

Fonte: http://architecturehabitat.blogspot.com.br/2010/10/finalsubmission.html

O método proposto para representar e analisar a geometria de superfície mínima

\section{Representação conceitual por curvas geratrizes e diretrizes e geração da superfície entre as curvas.}

Visando compreender conceitualmente os elementos geradores da superfície da obra, foi desenvolvido um modelo aproximado desta a partir da representação de curvas e geração da superfície por varredura e interpolação das curvas. Para tanto, inicialmente buscaram-se imagens da cobertura da obra e informações relevantes, tais como: se havia alguma descrição explícita dos tipos de curvas envolvidas nesta geometria; e se havia alguma referência a modelos digitais desenvolvidos para identificar os possíveis processos de geração da superfície.

Nesta busca de informações, ao procurar-se na Internet pelas palavras-chave '3D model Main Station', encontrouse a página que continha as informações e as imagens do modelo de Elementos Finitos desenvolvido pela empresa ROK. Tais imagens possuiam a vista superior do módulo básico que se repete ao longo da estrutura (hexagono marcado em cinza na parte inferior à esquerda da Figura 7). As imagens em perspectiva auxiliaram a compreender as curvas e o processo de geração da superfície e as apresentadas em Burry e Burry [11] e no site de Ingenhoven Architects possuíam as vistas ortográficas do projeto, sobre as quais foi possível mapear graficamente as curvas geratrizes (imagem com fundo preto na Figura 7).

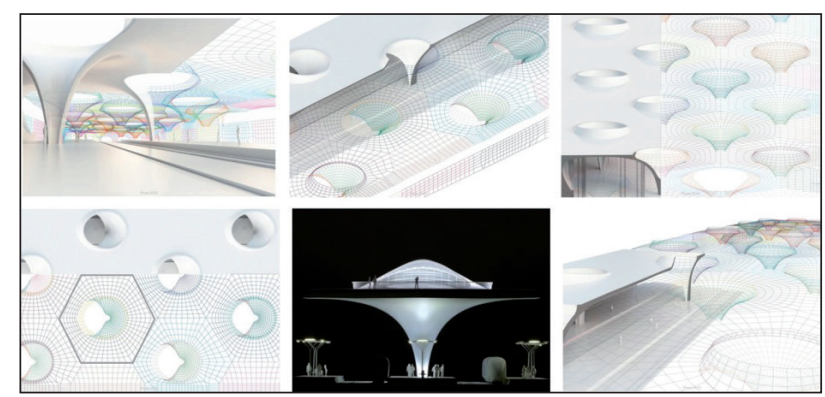

Figura 7: Imagens do projeto da cobertura do Main Station Stuttgart. Fonte: ROK (http://www.rok-office.com/projects/0990-stuttgart-21-parametric-fem/) e Ingenhoven Architects (http://www.ingenhovenarchitects.com/projects/more-projects/main-station-stuttgart/) 
A análise sobre as imagens em vista superior, vistas ortográficas e perspectivas foi essencial para identificar e delinear graficamente o módulo básico estrutural e as geratrizes e diretrizes que geram as superfícies. Identificaramse duas porções distintas de superfícies: uma superfície uniforme (superfície 1), na qual é possível distinguir um único tipo de curva geratriz em sua geometria, e uma superfície com dois tipos de curvas geratrizes (superfície 2), apoiadas em diretrizes circulares (Figura 8).
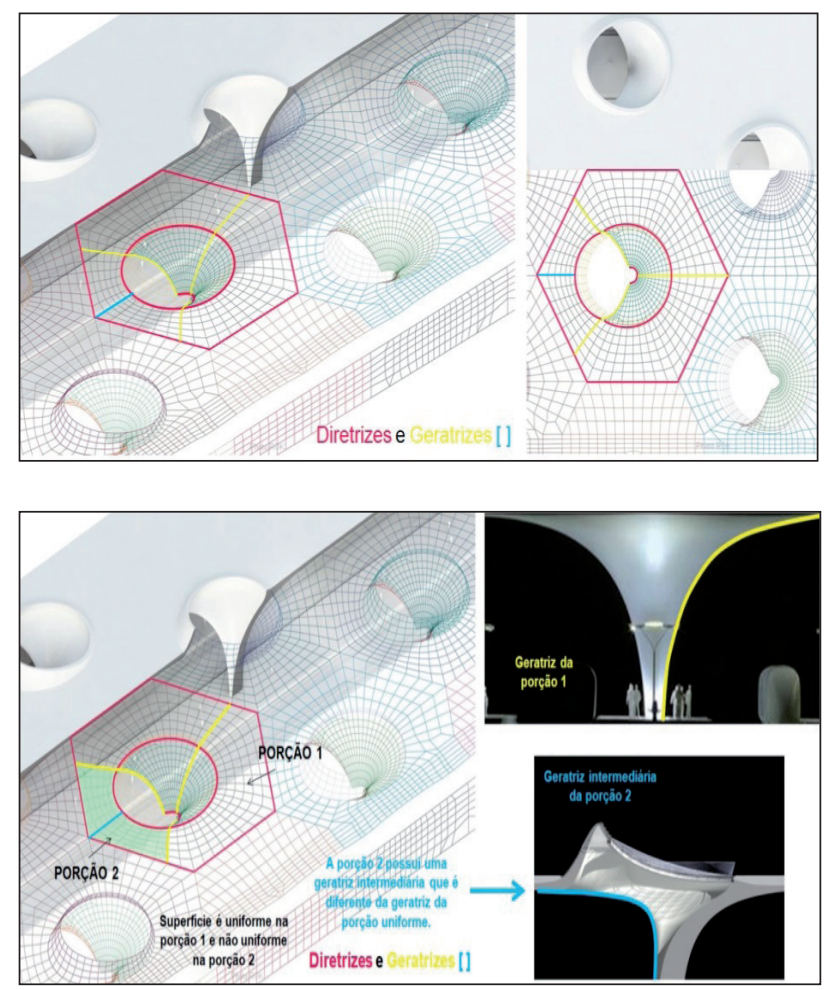

Figura 8: Análise geométrica: identificação das geratrizes e diretrizes da superfície do Main Station Stuttgart.

Fonte: Elaboração própria a partir das imagens de ROK (http://www. rok-office.com/projects/0990-stuttgart-21-parametric-fem/)

A análise sobre imagens da obra, em vista superior e frontal, também teve o objetivo de identificar as circunferências diretrizes da superfície (em vermelho), o ângulo definido nos arcos de circunferência sobre o qual ocorre o movimento circular das curvas geratrizes e as curvas geratrizes (em amarelo e azul nas mesmas Figuras). As alturas dos arcos diretrizes ficam definidas pelos pontos inicial e final das curvas geratrizes.

A partir destas análises foi possível delimitar um algoritmo de modelagem paramétrica, para cada uma das superfícies: A superfície 1 pode ser gerada pela varredura de uma única curva sobre a diretriz circular (processo de revolução); e a superfície 2 gerada por duas geratrizes curvas apoiadas em uma diretriz circular (varredura de curvas interpoladas ao longo de um arco). Ambas as superfícies são delimitadas por um contorno hexagonal (polígono de seis lados), o que pode ser obtido por seções sobre as superfícies.
O algoritmo correspondente a superfície 1 e o processo de modelagem paramétrica estão ilustrados na Figura 9, considerando-se 04 etapas de desenvolvimento: a representação da curva geratriz; a revolução da curva em torno de um eixo que passa pelo centro da superfície; a representação do hexágono de limite superior; e a seção da superfície pelo hexágono.

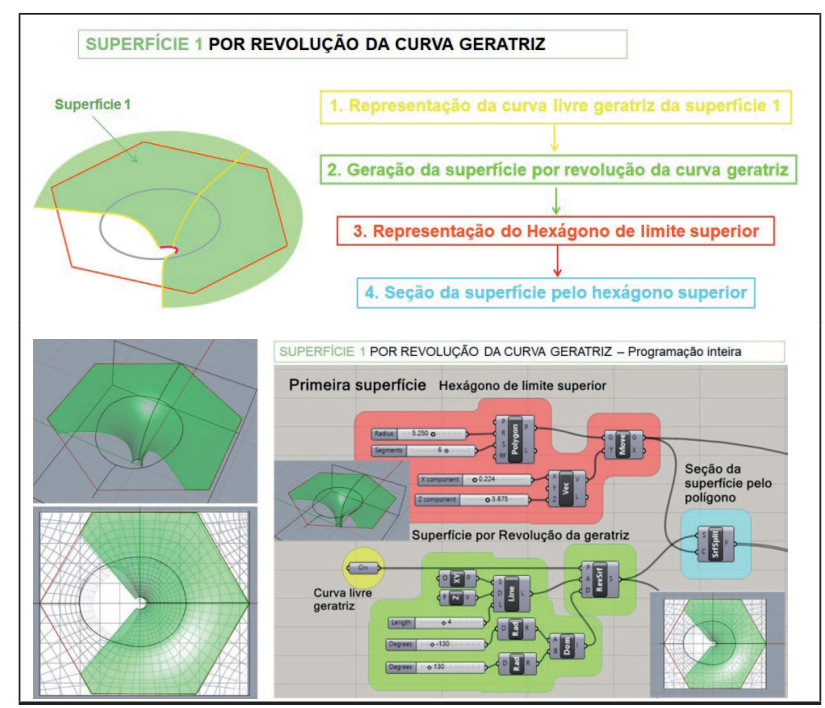

Figura 9: Algoritmo e modelagem paramétrica da superfície 1 do módulo básico da cobertura do Main Station Stuttgart.

Fonte: Elaboração própria.

A superfície 2 é definida por duas geratrizes iguais localizadas em simetria polar (rotação em torno do ponto central da superfície) e uma geratriz de configuração diferente, que se encontra na posição intermediária das geratrizes anteriores. As geratrizes extremas podem ser obtidas por extração das geratrizes que configuram a borda da superfície 1, sendo porções destas curvas. A geratriz intermediária pode ser obtida pelo desenho diretamente sobre a imagem da obra e a leitura de seus pontos de controle no ambiente de modelagem paramétrica, com posterior representação de uma curva livre interpolada por tais pontos.

$\mathrm{O}$ algoritmo com as etapas de representação das curvas e a geração da superfície, assim como o processo de modelagem paramétrica estão ilustrados na Figura 10, considerando-se 6 etapas para o seu desenvolvimento.

Para gerar a totalidade da estrutura foi aplicado um processo compositivo por simetrias de translação do módulo básico em x e y (Move) e por simetrias múltiplas em x e y dos módulos resultantes da primeira translação, as quais são representadas por meio de 'matrizes lineares' (Linear array). A composição final é obtida por aplicação de translações simultâneas em x e y dos dois módulos.

A Figura 11 ilustra um esquema gráfico e a programação visual destas etapas de modelagem paramétrica.

A Figura 12 apresenta as imagens do modelo paramétrico resultante, em vista superior e em perspectivas, com tomadas em aproximações do ponto de vista visando facilitar a percepção da geometria do modelo final obtido. 


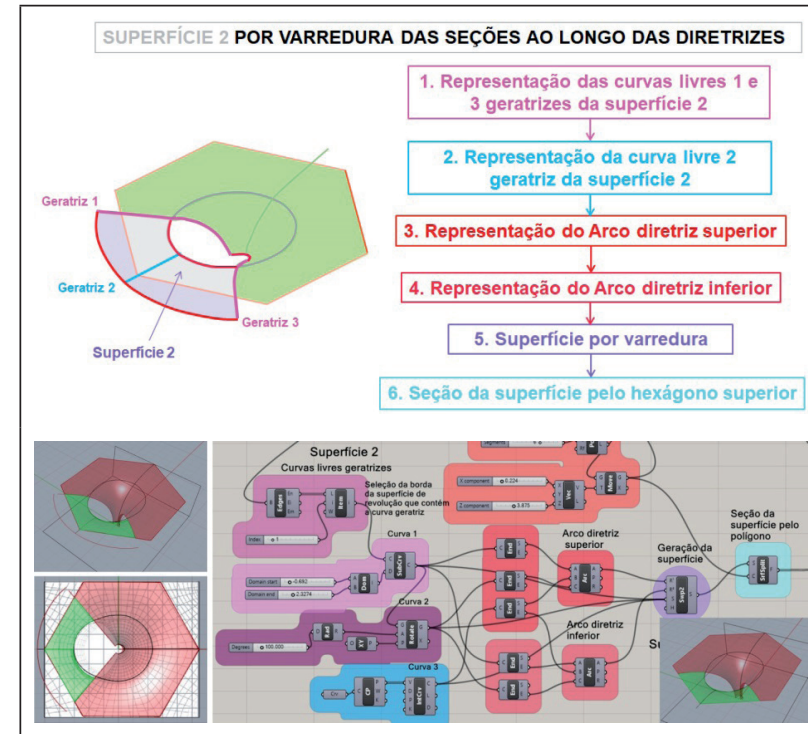

Figura 10: Algoritmo das etapas de modelagem paramétrica da superfície 2 da cobertura do Main Station Stuttgart e correspondente modelagem paramétrica.

Fonte: Elaboração própria.
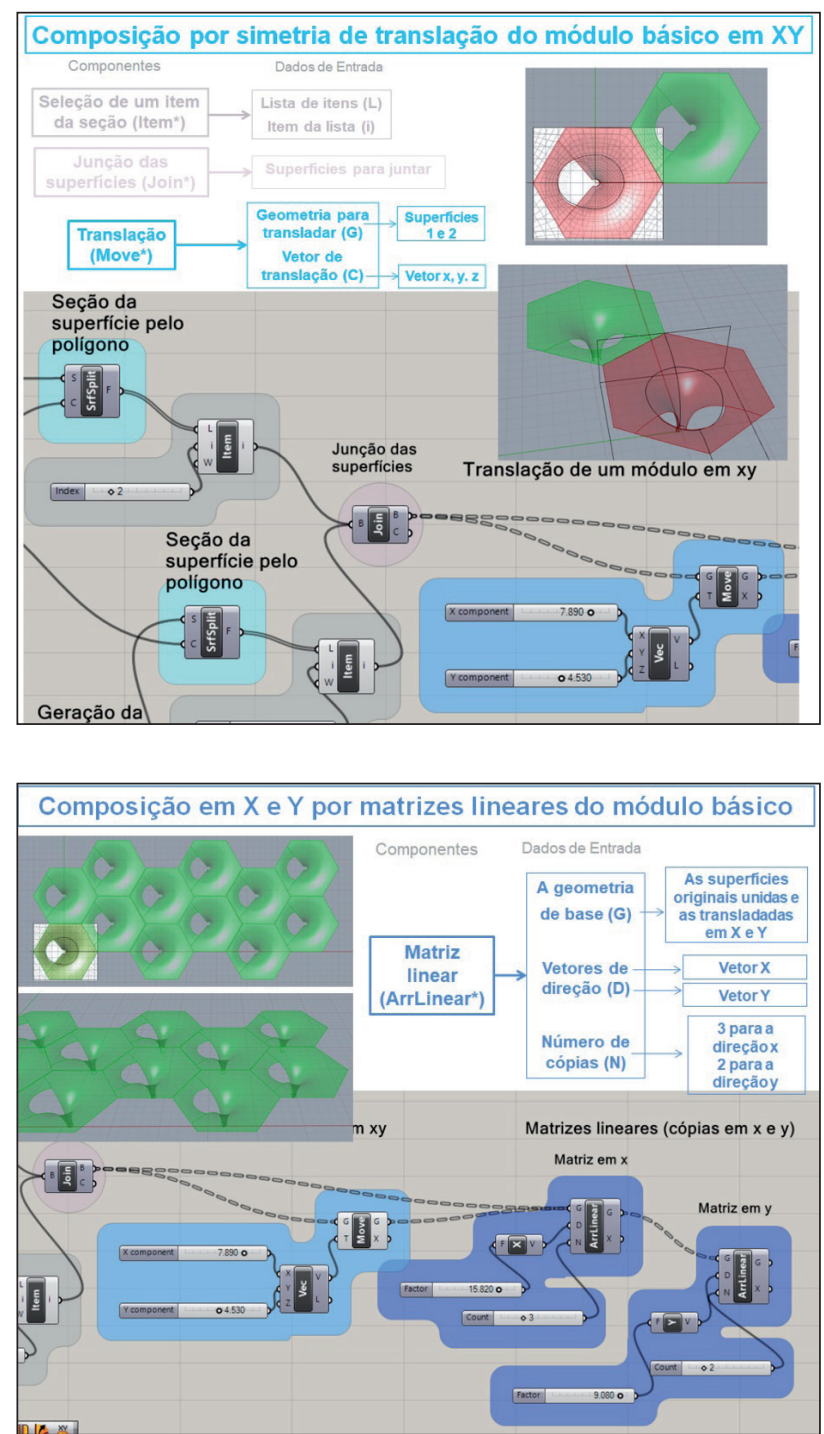

Figura 11: Algoritmo da etapa de modelagem paramétrica da simetria de translação da cobertura do Main Station Stuttgart e das simetria lineares para compor a totalidade da estrutura. Fonte: Elaboração própria.
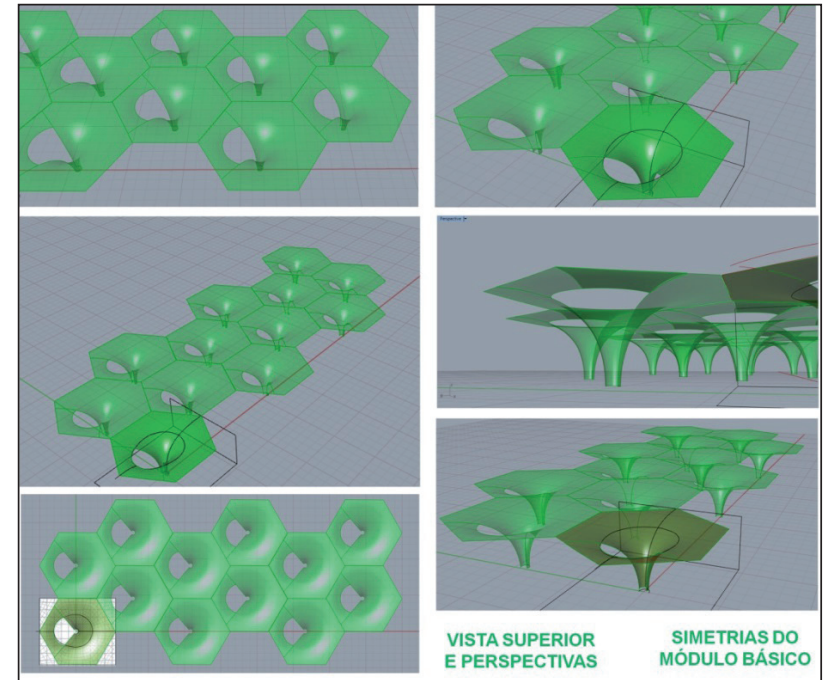

Figura 12: Modelo final paramétrico da cobertura do Main Station Stuttgart.

Fonte: Elaboração própria.

A verificação das curvaturas da superfície pode ser feita com o componente de análise de curvatura disponível no ambiente de programação visual, para avaliar o comportamento que caracteriza uma superfície mínima, dado pela curvatura média $(\mathrm{M})$ igual à zero em todos os pontos da superfície. Uma maneira mais intuitiva é avaliar a curvatura diretamente no software Rhinoceros, após exportar a geometria da programação para o ambiente deste software.

Na Figura 13 é possível observar a presença de curvaturas variáveis e poucos pontos com curvatura média igual a zero absoluto, os quais ocorrem próximos à região planar da superfície. Isto se deve a não precisão do modelo digital em relação ao modelo real do Main Station Stuttgart, o qual foi obtido por meio de experimentações físicas.

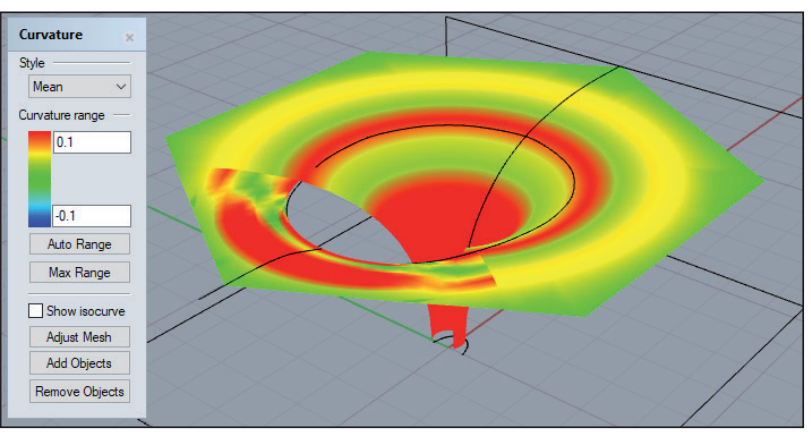

Figura 13: Análise de curvatura do modelo paramétrico da cobertura do Main Station Stuttgart.

Fonte: Elaboração própria.

Explorações adicionais podem ser feitas como a definição precisa das curvas geratrizes das superfícies 1 e 2 . No entanto, tais explorações exigem um reconhecimento específico dos tipos de curvas que conformam tal superfície. De outro modo, modelos obtidos a partir de simulações físicas, executadas empregando-se plug-ins que se integram à programação visual, podem auxiliar na análise, mapeamento e extração de tais curvas para um estudo mais 
aprofundado e a representação fidedigna de tal superfície.

Tais estudos não fazem parte do escopo deste trabalho, mas experimentos nesta direção já estão em curso no âmbito da pesquisa, com o propósito de avançar e ampliar os resultados obtidos até então.

\section{A estruturação do saber}

\section{A estrutura do conteúdo}

Com o propósito de sistematizar o saber envolvido em tal superfície, a estrutura de saber relativa ao conteúdo foi organizada a partir dos conceitos mais gerais para os conceitos particulares, incluindo duas classes principais: geometria e modelagem paramétrica. (Figura 14).

Esta estrutura se organiza em torno da(s): descrições apresentadas por Burry e Burry [11] para a obra e sua superfície geométrica; descrições dos mesmos autores para os conceitos de Superfície Mínima e Modelos de Catenária; imagens da obra disponibilizadas pelo escritório do arquiteto e por Burry e Burry [11]; ampliação das estruturas de saber a partir dos conceitos geométricos e da arquitetura relacionados com a superfície da obra e a partir da modelagem paramétrica de tal superfície. Estes blocos integram o primeiro nível da estrutura da rede de conceitos.

\section{A ampliação das estruturas de saber para a didática em arquitetura}

A partir do método adotado, reconheceu-se o conceito de 'modelos de catenária' e de 'modelos funiculares' e o histórico da evolução destes conceitos em sua aplicação na arquitetura. $\mathrm{Na}$ estrutura de conceitos proposta, estes temas foram considerados como fundamentos geométricos da superfície em questão, porque as estruturas de saber que foram explicitadas explicam como se dá o funcionamento estrutural de tais modelos e a lógica de seu desempenho funcional na configuração de geometrias com características de superfícies mínimas. Também foram inseridas estruturas de saber específicas sobre a teoria de superfícies mínimas, porque estas estão relacionadas com a caracterização dos modelos funiculares em geral. A associação entre os conceitos também é importante de um ponto de vista geométrico, pela abordagem matemática relativa à característica da curvatura destas superfícies.

Outra abordagem importante e que foi considerada nesta ampliação de estruturas de saber para o ensino de Arquitetura é da Arquitetura regenerativa, que possui associação direta com a proposição de modelos geométricos voltados ao desempenho, imitando as funcionalidades dos modelos da naturaza. Estruturas concebidas nesta abordagem e complementadas com materiais e sistemas construtivos que possibilitem obter uma relação de equilíbrio energético com o meio ambiente em que se inserem, têm grande potencial para avançar em concepções regenerativas na arquitetura.

Na caratcterização da geometria do modelo funicular da estação de trem estão incluídos elementos de saber que possibilitam compreendê-la de maneira mais explícita, de modo a facilitar a modelagem paramétrica. Esta por sua vez se fundamenta na (o): análise da superfície, sua caracterização quanto aos tipos de curvas geratrizes e diretrizes e a identificação de seus parâmetros geométricos, sua descrição gráfica e o algoritmo de modelagem, e, por fim,

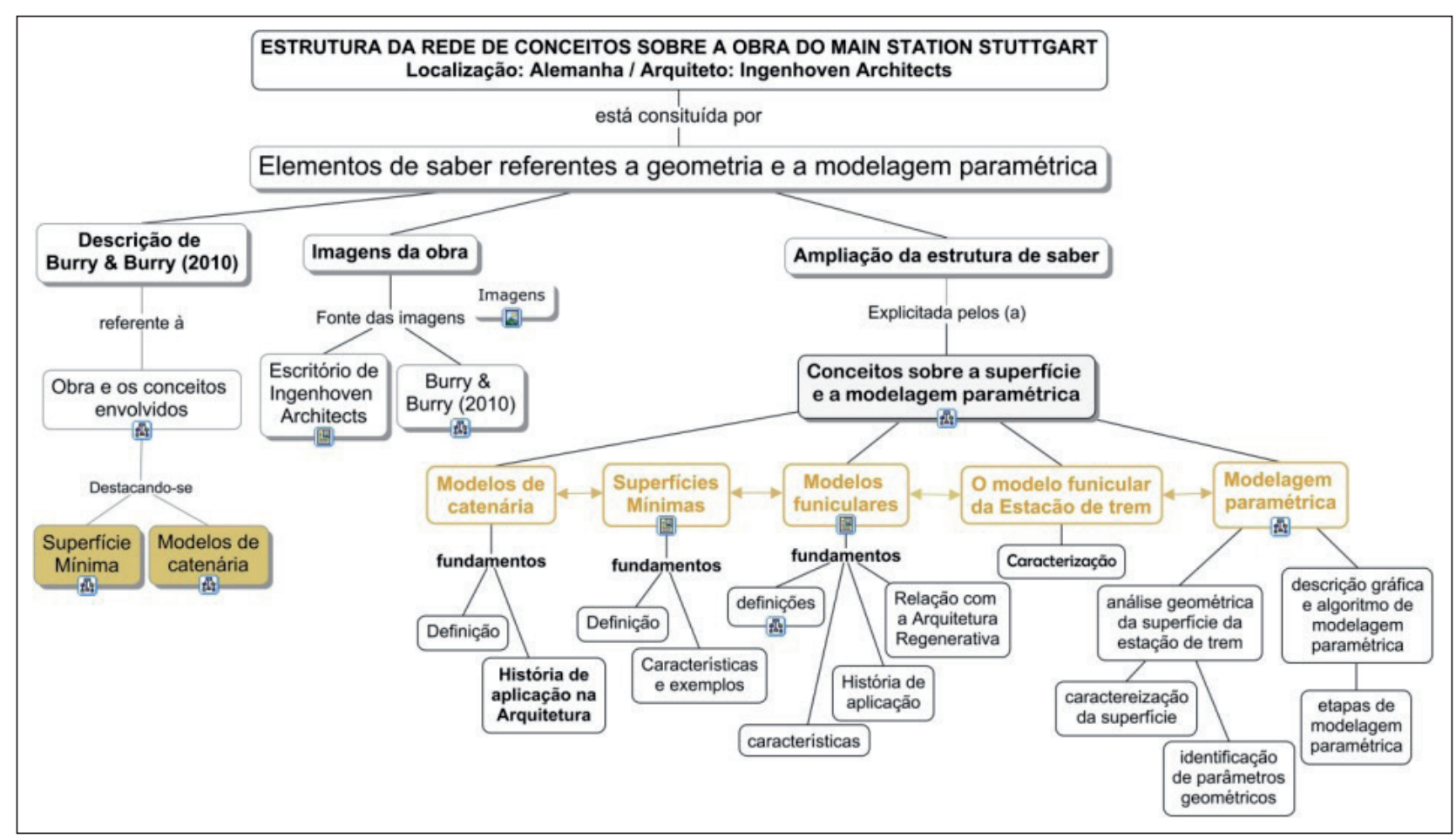

Figura 14: A estrutura da rede de conceitos da geometria e modelagem paramétrica da estação de trem de Stuttgart. Fonte: Elaboração própria. 
delineamento de suas etapas. A rede completa de conceitos está disponível em (retirado para a revisão às cegas).

\section{Resultados e Discussão}

A geometria funicular reconhecida neste trabalho é notadamente utilizada na arquitetura em processos projetuais que buscam a otimização estrutural da forma a ser construída como também para alcançar funcionalidades e estética. Os problemas de otimização, em uma perspectiva de design que integre estética e requisitos funcionais, pertencem a uma área problemática, difícil e complexa [13]. O que pode estar relacionado com o fato de algumas geometrias complexas da arquitetura contemporânea ser desenvolvidas com um apelo exclusivamente formal e estético.

Aliar a estética, a forma, o espaço e a função em uma proposição arquitetônica global e coerente foi o objetivo dos arquitetos do passado, citados anteriormente, e outros tais como Frank Lloyd Wright, Louis Henri Sullivan e Rudolf Steiner. As estratégias holísticas prosperaram nesse sentido com inspiração tanto nas formas naturais como em processos naturais [18]. Especialmente por meio das estruturas de tração e das conchas finas de concreto armado Frei Otto e Felix Candela, extraíram conceitos de construção e de estrutura, inerentes aos princípios naturais e biológicos.

Os modelos experimentais baseados no funcionamento das estruturas da natureza (modelos funiculares ou de superfícies mínimas) de Gaudi e Frei Otto trouxeram novos desenvolvimentos para arquitetura, influenciando arquitetos do presente e oferecendo possibilidades futuras. A arquitetura contemporânea apresenta exemplos do emprego destes modelos, principalmente quando o foco é a 'arquitetura regenerativa' [19].

Identifica-se desta maneira, a necessidade de, no contexto educativo de arquitetura, tratar da relação entre forma e estrutura a partir do conhecimento e experimentação com modelos funiculares desenvolvidos ao longo da história, também denominados de 'modelos de construção'.

D'Arcy Thompson foi o primeiro a desenvolver pesquisas na área denominada de morfologia estrutural, definida como o estudo da forma em relação às vias de forças que transitam nos elementos estruturais, os quais materializam o fluxo de forças [20]. A forma, assim vista como consequência de deformações físicas, definida por leis e forças naturais, como tensão superfícial ou gravidade, princípio que é ainda hoje aplicado pelos arquitetos. O modelo de forma/forças pode ser entendido como 'modelos renovados de conhecimento' e do processo de design, em nível cognitivo e operacional, os quais evoluíram para modelos construtivos e experimentais [18].

A compreensão da arquitetura contemporânea sob o aspecto morfológico pode trazer uma reconciliação entre a forma e estrutura, a construtibilidade, modelos cognitivos, operacionais e experimentais, além de conceitos geométricos e arquitetônicos importantes de serem tratados no contexto de formação em arquitetura. Dessa maneira, a didática arquitetônica pode ser favorecida ao tratar de modelos geométricos funiculares que possuam processos de geração conhecidos, em detrimento ao emprego de formas livremente obtidas com ausência de propriedades de desempenho arquitetônico.

Haveria um ganho consideravelmente qualitativo pelas propriedades funcionais particulares que estes modelos apresentam. A questão é que, no ensino de arquitetura, é dado muito mais interesse aos processos intuitivos de obtenção das formas do que ao estudo sobre a geometria que as define como conhecimento de projeto.

Se por um lado, o estudo possibilitou compreender em um nível mais profundo o tipo de superfície adotada na estação de trem de Stuttgart, por outro lado, a sistematização de processos de modelagem paramétrica das geometrias associadas às estruturas naturais, exigiu identificar estruturas de saber relacionadas às teorias e técnicas que possibilitam representar as geometrias de superfícies mínimas. As teorias trouxeram novas estruturas de saber para uma maior compreensão de tais superfícies, principalmente no âmbito da matemática e geometria, da representação gráfica digital e do ensino de arquitetura.

Conforme mencionado no referencial teórico, a explicitação de tais estruturas de saber é essencial para fundamentar o desenho de situações didáticas para arquitetura com foco na geometria complexa e em sua modelagem paramétrica. Além disso, possibilitam aos estudantes terem uma maior compreensão dos processos de geração de tais geometrias e das ações projetuais dos arquitetos que as propõem em seus edifícios, vindo a refletir sobre seus próprios processos projetuais de maneira consciente.

$\mathrm{O}$ reconhecimento de tais estruturas de saber apontou também a necessidade de detalhar em um nível mais profundo algumas caracterizações apresentadas pelos autores de referência. Principalmente quanto aos tipos de curvas diretrizes e geratrizes de tais superfícies e os processos compositivos que estas possuem. Embora as representações propostas não abordem saberes mais específicos do cálculo matemático e da física, os quais envolveriam álgebra, funções ou descrição paramétrica de curvas, e a lógica de programação, a explicitação geométrica de tais superfícies trouxe o reconhecimento de seus entes geométricos elementares.

\section{Conclusão}

A partir do estudo desenvolvido, foi possível compreender que o conhecimento sobre superfícies mínimas, especialmente as configuradas por modelos físicos, identificadas como recorrentes em exemplares da arquitetura contemporânea, configura-se uma estratégia didática importante para o ensino de projeto atual. Isto se deve a estas estarem estritamente relacionadas ao funcionamento das estruturas encontradas na natureza, permitindo aprender so- 
bre a morfologia ótima, a integração funcional e eficiência, atributos relacionados com a sua geometria [18].

Pottmann et al. [13] destaca que a realização de uma ideia de design de forma ótima é uma tarefa difícil e complexa, principalmente por sua formulação matemática e algorítmica, ainda mais tendo que levar em conta requisitos funcionais. $\mathrm{O}$ autor reitera que as abordagens centrada em curvas, superfícies e malhas muito bem conhecidas podem ser tomadas como possíveis soluções de problemas de otimização, os quais são associados diretamente às superfícies mínimas, merecendo atenção, de uma perspectiva arquitetônica. Além da abordagem de curvas e superfícies, os modelos físicos que conformam superfícies mínimas ao serem estudados nesta perspectiva integrados a simulações digitais paramétricas por atuação de forças, permitem tratar com a relação entre forma e estrutura.

Como pesquisa futura e que se encontra em desenvolvimento no presente momento é ampliar o desenho de situações didáticas para além dos modelos aproximados de superfícies mínimas, buscando-se representar tais geometrias por meio de modelos geométricos fidedignos integrados à construção de modelos físicos em suspensão. Estas atividades serão experimentadas em contextos de ensino do curso de Arquitetura e Urbanismo, na Universidade (retirado para não identificação), e posteriormente avaliadas quando aos objetivos didáticos traçados.

A modelagem geométrica dos modelos em suspensão está sendo obtida por meio de simulações digitais que integram os tipos de forças físicas a que tais superfícies ficam submetidas e as propriedades do material. As etapas de simulação digital destas condições e de conformação do modelo geométrico da obra Main Station Stuttgart (que utiliza um modelo de corrente suspensa de Frei Otto) já estão concluídas, sendo que a próxima etapa da pesquisa é a de estruturar e aplicar as atividades didáticas que visam tal integração entre simulação e construção física, aplicadas ao projeto de arquitetura.

Em relação aos aspectos didáticos, visando à inserção no ensino de arquitetura, considera-se que a explicitação das teorias e técnicas de modelagem paramétrica de tais geometrias provém uma base teórica e tecnológica de fundamentos para a concepção da arquitetura direcionada as abordagens contemporâneas de design.

\section{Agradecimentos}

Agradecemos a Universidade [retirado para revisão às cegas] pela oportunidade de desenvolver esta pesquisa em nível de doutoramento e ao CNPQ por apoiar o desenvolvimento da rede [retirado para revisão as cegas], a qual oportunizou a disponibilização da rede de conceitos associada a esta pesquisa.

\section{Referências}

1. Bill, A. Edificação: 3000 Anos de Projeto, Engenharia e Construção. Porto Alegre: Bookman, 2009. Tradução de Alexandre Salvaterra.

2. Perez-Garcia, A.; Gómez-Martínez, F. Natural structures: strategies for geometric and morphological optimization. Proceedings of the International Association for Shell and Spatial Structures (IASS) Symposium 2009, Valencia Evolution and Trends in Design, Analysis and Construction of Shell and Spatial Structures 28 September - 2 October 2009, Universidad Politecnica de Valencia, Spain. Alberto DOMINGO and Carlos LAZARO (eds.)

3. Rippmann, M. Block, P. Funicular Shell Design Exploration. Acadia 2013, Adaptive Architecture. 2013.

4. Retirado para a revisão às cegas.

5. Chevallard, y. El Análisis de las Prácticas Docentes en la Teoría Antropológica de Lo Didáctico. Recherches en Didactique de Mathématiques, Grenoble, Vol. 19, n 2, pp. 221-266, 1999. (Traducción de Ricardo Barroso, Universidad de Sevilla). Disponível em: $<$ http://www. aloj.us.es/rbarroso/Pruebas/CHEVALLARD.PDF>

6. Woodbury, R. Elements of Parametric Design. London: Routledge, 2010.

7. Carmo, M. P. Superfícies Mínimas. Rio de Janeiro: Instituto de Matemática Pura e Aplicada - IMPA, 1987.

8. Mark, E. Martens, B. Oxman, R. Preliminary stages of CAAD education. Automation in Construction 12 (2003) 661670662.

9. Kvan, T. Mark, E. Oxman. R. Martens, B. Ditching the Dinosaur: Redefining the Role of Digital Media in Education. Disponível em: http:/www-2016.arch.virginia. edu/arch541/dinosaur.html Acesso em: dezembro 2016.

10. Chevallard, y. La Transposición didáctica. Del saber sabio al saber enseñado. Buenos Aires: Aique Grupo Editor, 2013. $3^{\mathrm{a}}$ ed. $4^{\mathrm{a}}$ reimp. Título original: La transposition didactique. Du savoir savant au savoir enseigné. Grenoble: Pensée Sauvage, 2ème édition, 1991.

11. Burry. J. Burry, M. The New Mathematics of Architecture. London: ed. Thames e Hudson, 2010.

12. Main Station Stuttgart: Global project posters 2006. Disponível em : https://src.lafargeholcim-foundation.org/dnl/ c8b222e5-9274-4e06-93d9-bf4a8e8dbd43/EU05_ OVGGG.pdf Acesso em: abril 2018.

13. Pottmann, H. Asperl, A. Hofer, M. Kilian, A. Architectural Geometry. Exton, Pennsylvania: Bentley Institute Press, $1^{\text {a }}$ ed., 2007.

14. Kanaiya, M. What is Funicular geometry? What is its significance in Structures in Architecture? Uses geometry in architecture. 2013. Disponível em: <https://www. quora.com/What-is-Funicular-geometry-What-is-itssignificance-in-Structures-in-Architecture> 
15. Huerta, s. Structural Design in the Work of Gaudi. Architectural Science Review. Volume 49.4, pp 324 - 339, 2006.

16. Chilton, J. The Engineer's Contribution to Contemporary Architecture: Heinz Isler. London: Thomas Telford Press, 2000.

17. Chilton, J. Chung, c. c. Rooted in Nature: Aesthetics, Geometry and Structure in the Shells of Heinz Isler. Nexus Network Journal, v. 19, issue 3, pp. 763-785, 2017.

18. Stals, A.; Elsen, C.; Jancart, S.; Delvaux, F. Challenges in Teaching Architectural Morphogenesis. Learning x Design - The 3rd International Conference for Design Education Researchers. 2015.

19. Littman, J. A. Regenerative Architecture: A Pathway Beyond Sustainability. Dissertação de Mestrado.
University of Massachusetts - Amherst. 2009. 68 p. Disponível em: <http://scholarworks.umass.edu/cgi/ viewcontent.cgi? article $=1389 \&$ context $=$ theses $>$ Acesso em: abril 2015.

20. Bertol, D. Form Geometry Structure: from nature to design. Exton, Pennsylvania: Bentley Institute Press, 2011. 